\section{Sobre la donación de órganos para trasplantes y la Atención Primaria}

\section{Sr. Director:}

Tras leer con interés la editorial sobre "La donación de órganos para trasplantes y la Atención Primaria" de Cuende y Miranda $^{1}$, en la que se revisa excelentemente la situación actual de la donación de órganos en nuestro país, creemos, sin embargo, que sólo trata tangencialmente el importante papel que la Atención Primaria puede jugar en beneficio de la donación de órganos.

Es indudable que la Atención Primaria ofrece una serie de ventajas en cuanto al acceso a la población y la posibilidad de influir con mensajes positivos hacia la donación de órganos ${ }^{2}$. Sin embargo, a pesar de esta situación teórica favorable debemos tener en cuenta que con frecuencia los centros de salud se encuentran saturados de actividad asistencial ${ }^{2}$, con un elevado número de pacientes por consulta, y múltiples programas sanitarios (hipertensión arterial, diabetes, etc.), lo que hace impensable en muchos casos el poder programar actividades en este sentido ${ }^{2}$. Además, es frecuente que muchos centros de salud tengan proyectos de otros programas asistenciales (control de alcohólicos, cuidados de colostomías, cuidados del pie diabético, etc.), que no han podido todavía iniciarse por falta de personal, lo que hace más difícil pensar en actuaciones de educación sanitaria.

En la Coordinación Regional de Trasplantes de nuestra comunidad se han integrado personas de Atención Primaria que están promoviendo un proyecto de educación sanitaria sobre donación de órganos ${ }^{3-7}$. Sin embargo esta colaboración viene determinada por el voluntarismo y una inquietud personal hacia dicho tema, por parte de estos médicos de familia, ya que estas actividades suponen una sobrecarga importante de trabajo extra-laboral no reconocido. Si queremos realmente que Atención Primaria se inte- gre en la promoción de la donación de órganos, será necesario diseñar un plan de actuación bien estructurado, siendo el primer paso y fundamental la realización de cursos informativos y formativos para el personal de Atención Primaria, pues es difícil informar bien de aquello que se desconoce o sólo se conoce parcialmente. Además sería preciso que la promoción de la donación se incorporara como una actividad más del centro de salud, como uno de los objetivos globales de educación sanitaria. Mientras no se desarrolle un plan general de actuación que implique a todos los centros de salud del Sistema Nacional de Salud, y nos quedemos sólo en buenas intenciones, no conseguiremos desde los centros de Atención Primaria ayudar a descender las negativas familiares a la donación de órganos en nuestro país.

\section{Conesa Bernal, A. Ríos Zambudio*, P. Ramírez Ro- mero**}


Médico de Medicina de Familia y Comunitaria. Centro de Salud de Fortuna. *Cirugía General y del Aparato Digestivo. Hospital Uni versitario Virgen de la Arrixa ca. **Coordinador Regional de Trasplantes de la Comunidad Autónoma de Murcia.

Centro de Salud de Fortuna. Murcia. Coordinación Regional de Trasplantes de la Comunidad Autónoma de Murcia.

1. Cuende N, Miranda B. La donación de órganos para trasplante y la Aten- ción Primaria. Medifam 2000; 10 143-7.

2. Ríos Zambudio A, Conesa Bernal C, Munuera Orenes C, Fajardo Motos D. Donación y trasplante de órganos en España: importancia del papel de la Atención Primaria. Medicina Integral 1999; 33: 89-95.

3. Ríos A, Murcia JA, Conesa C, Munuera C, Pascual FJ, Fajardo D. Situación del conocimiento sobre donación y trasplante de órganos en escolares de la huerta murciana. Salud Rural 1997; 14: 83-90.

4. Ríos A, Murcia JA, Conesa C, Munuera C. ¿Tienen más conocimientos sobre donación y trasplante de órganos los escolares de ámbito religioso que los de ámbito laico? Semergen 1999; 25: 358-60.

5. Ríos A, Murcia JA, Conesa C, Munuera C. ¿Qué conocimientos tienen los niños murcianos sobre la donación y trasplante de órganos? Estudio preliminar. Aten Primaria 1998; 21: 116-7.

6. Ríos A, Conesa C, Munuera C, Pascual FJ, Fajardo D. Campaña de concienciación sobre la donación y trasplante de órganos en la Comunidad Autónoma de Murcia. Estudio piloto. Semergen 1999; 25: 495-7.

7. Ríos A, Conesa C, Munuera C, Pascual FJ, Fajardo D. Campaña informativa sobre la donación y trasplante de órganos en escolares. Aten Primaria 1998; 21: 623-6. 